\title{
Kapitel 8 \\ Praktiken zur Steuerung und Kontrolle von \\ Contract-Manufacturing-Dienstleistern in der Supply Chain
}

\section{Konzeptionelle Überlegungen und erste empirische Befunde am Beispiel der pharmazeutischen Industrie}

\section{Wolfgang Stölzle und Tim Brandl}

Zusammenfassung Markenführende Unternehmen, die Teile ihrer Fertigungsaktivitäten an Contract-Manufacturing-(CM-)Dienstleister vergeben, stehen vor der Herausforderung, die termin- und qualitätsgerechte Produktherstellung trotz eingeschränkten Zugriffs auf die Fertigungsprozesse sicherzustellen. Doch die Produktion durch einen CM-Dienstleister hat für outsourcende Unternehmen häufig die Charakteristika einer „Black Box“. Vor diesem Hintergrund sind solche Unternehmen aufgerufen, geeignete Management-Praktiken zur Steuerung und Kontrolle der CM-Dienstleister einzusetzen. Aufgrund der hohen Individualität von Contract-Manufacturing-Vereinbarungen gestaltet sich die systematische Auswahl geeigneter Praktiken komplex. Dieser Beitrag erforscht, mit welchen Praktiken Unternehmen ihre CM-Dienstleister steuern und kontrollieren können, um ihre mit der Fremdvergabe verbundenen Ziele zu erreichen. $\mathrm{Zu}$ diesem Zweck werden Erkenntnisse aus der Literatur analysiert und durch empirische Befunde aus sechs Fallstudien mit explorativem Charakter von Unternehmen der pharmazeutischen Industrie ergänzt. Basierend auf den Untersuchungsergebnissen der Fallstudien werden erste Erkenntnisse und weiterer Forschungsbedarf für das Management von CM-Dienstleistern in der Supply Chain entwickelt.

\author{
W. Stölzle $(\bowtie)$ \\ Universität St.Gallen (HSG), St. Gallen, Schweiz \\ E-Mail: wolfgang.stoelzle@unisg.ch \\ T. Brandl \\ Universität St.Gallen (HSG), St. Gallen, Schweiz
}




\subsection{Contract Manufacturing im Lichte des Supply Chain Managements}

Die Wertschöpfungstiefe produzierender Unternehmen hat sich in den letzten Jahrzehnten branchenübergreifend verringert. Der Wertschöpfungsanteil von CMDienstleistern beträgt mitunter über $50 \%$ der Gesamtherstellkosten (Tsay et al. 2018). In der Elektronikindustrie (siehe Zion Market Research 2019) und in der Pharmabranche (siehe Grand View Research) wird in den nächsten Jahren von einem weiter anhaltenden, dynamischen Wachstum des Contract Manufacturing ausgegangen. Vor diesem Hintergrund stehen Unternehmen zunehmend vor der Herausforderung, die Erreichung operativer Zielkenngrößen wie Kosten, Qualität und Termintreue in ihrer Supply Chain ohne direkten Zugriff auf die fremdvergebenen Fertigungsprozesse zu gewährleisten. Untersuchungen zufolge werden im verarbeitenden Gewerbe in Deutschland zwischen $15 \%$ und $25 \%$ aller Fremdvergabe-Entscheidungen innerhalb von zwei bis fünf Jahren zurückgenommen (Kinkel 2014). In mehr als $80 \%$ der Fälle werden die Fertigungsaktivitäten ins Unternehmen reintegriert, da die angestrebten Qualitäts- und Kostenziele im Rahmen der Fremdvergabe nicht erreicht werden (Kinkel 2014; Foerstl et al. 2016). Daher ist es notwendig, dass Unternehmen die Erreichung operativer Zielkenngrößen im Zusammenhang mit den fremdvergebenen Fertigungsaktivitäten systematisch überwachen. Durch die Fremdvergabe entziehen sich die Fertigungsprozesse dem direkten Zugriff des Unternehmens. Folglich müssen sich Mechanismen zur Steuerung und Kontrolle der Kenngrößen auf die CM-Dienstleister beziehen. In der Praxis wird in diesem Zusammenhang von Praktiken zum Management der CM-Dienstleister gesprochen.

Das Management von CM-Dienstleistern zeichnet sich durch eine hohe Komplexität infolge der kundenindividuellen Erbringung der Dienstleistung und des oft hohen Grads der Integration des Dienstleisters in die eigenen Geschäftsprozesse aus. Die Einhaltung kundenspezifischer Prozessvorgaben und das Risiko potenzieller Reputationsschäden erfordern über die Bewertung des fertigen Produkts hinaus Mechanismen zur Überwachung der Fertigungsprozesse beim Dienstleister (Kang et al. 2012). Die Anforderungen an die Steuerung und Kontrolle der CM-Dienstleister unterscheiden sich in Abhängigkeit der Zielvorgaben und des Risikoprofils der fremdvergebenen Fertigungsprozesse erheblich und erfordern einen situativen Management-Ansatz (Kang et al. 2014; van Weele und Essig 2017). Durch die Integration des CM-Dienstleisters in die Fertigung des eigenen Produkts entsteht entlang des Fertigungsprozesses eine Vielzahl Schnittstellen zwischen unternehmensinternen Bereichen wie Einkauf oder Produktion einerseits und dem CM-Dienstleister andererseits. Daraus ergibt sich die Frage nach der organisatorischen Zuständigkeit für die Anwendung von ManagementPraktiken. Trotz der Herausforderungen bei der Steuerung und Kontrolle fremdvergebener Fertigungsprozesse sind das Vorgehen und die Anwendung spezifischer Management-Praktiken von Unternehmen in der Praxis weitestgehend unerforscht. Als Grundlage für die Analyse, warum eine hohe Anzahl an Fremdvergaben 
von Fertigungsprozessen scheitert, betrachtet dieser Beitrag den Status Quo der Steuerung und Kontrolle von CM-Dienstleistern in der Praxis.

Damit geht es um die Fragestellung, welche Steuerungs- und Kontrollmechanismen in Form von Management-Praktiken von Unternehmen eingesetzt werden (Forschungsfrage 1). Darauf aufbauend sind der Kontingenztheorie folgend relevante Kontextfaktoren für die Anwendung der erhobenen Management-Praktiken zu identifizieren (Forschungsfrage 2). Daraus ergibt sich die Frage, wie im Lichte der oft hohen Anzahl an Schnittstellen zum CM-Dienstleister die interne Zuständigkeit für die Anwendung von Management-Praktiken im Unternehmen zu organisieren ist (Forschungsfrage 3).

$\mathrm{Zu}$ diesem Zweck wird zunächst ein Überblick über den Stand der Forschung zur Einbindung von CM-Dienstleistern gegeben und es werden die Forschungslücken spezifiziert. Ein Denkrahmen dient dazu, die Forschungsfragen anhand der relevanten Forschungslücken und des Praxisbedarfs abzuleiten. Zur Beantwortung der Forschungsfragen werden in einem explorativen Fallstudienansatz Primärdaten aus sechs Unternehmen der pharmazeutischen Industrie, die Contract Manufacturer einbinden, gewonnen. Auf Basis der Analyse der Fallstudien werden Muster identifiziert, um ein erstes Verständnis für die Steuerung und Kontrolle von CM-Dienstleistern durch fremdvergebende Unternehmen zu gewinnen. Die Beantwortung der Forschungsfragen liefert Implikationen für die Einbindung von Contract Manufacturern in den Wertschöpfungsprozess und dient als Grundlage für weitere Forschungsaktivitäten zum Contract Manufacturing im Supply Chain Management.

\subsection{Wissenschaftliche Perspektive zum Management von Contract-Manufacturing-Dienstleistern}

Das Phänomen des Contract Manufacturing ist in der Forschung sehr präsent und wird in unterschiedlichen Forschungsströmungen betrachtet.

\subsubsection{Forschungsströmungen und aktueller Forschungstand}

Die Forschung zum Contract Manufacturing hat sich seit den 1970er Jahren in der Literatur etabliert und wird in verschiedenen Forschungsströmungen konsequent vorangetrieben (Cavinato 1989). Für das Management von CM-Dienstleistern ist sowohl die Forschung zu konkreten Management-Ansätzen als auch zu Faktoren von Interesse, welche den Anwendungskontext von Management-Praktiken beeinflussen.

Der Begriff „Management-Praktiken“ ist eine in der Praxis gebräuchliche Bezeichnung und lehnt sich inhaltlich an die englische Bezeichnung „Organizational 
Control Practices“ an (Kang et al. 2012). Management-Praktiken sind spezifische Methoden, welche in einem Geschäftsprozess durch Organisationseinheiten angewendet werden, um andere Organisationseinheiten gezielt zu beeinflussen und sicherzustellen, dass bestimmte Zielkenngrößen erreicht werden (Kang et al. 2012).

Der Anwendungskontext von Management-Praktiken ist eng mit den Leistungszielen verknüpft, welche durch das Contract Manufacturing angestrebt werden. Ausgehend von der Senkung von Transaktionskosten als primärem Leistungsziel für Contract Manufacturing (Serel et al. 2001; Kim 2003) sind in den folgenden Jahren vor allem die Verbesserung nicht-monetärer Leistungsgrößen, wie der Flexibilität (Mason et al. 2002; Oke 2005; Mohammed et al. 2008; Soon und Udin 2011), der Innovationsfähigkeit (Oke und Onwuegbuzie 2013; Oduro 2019) sowie individuell zielgerichteter Supply Chain Designs (Singh Srai und Gregory 2008; Campagnolo und Camuffo 2017) in den Fokus gerückt. Die Forschung konnte den Einfluss des Contract Manufacturing auf diese Eigenschaften nachweisen und ein größeres Verständnis für die mit dem Contract Manufacturing verbundenen Zielgrößen schaffen.

Damit einher geht der Wandel der Wahrnehmung des CM-Dienstleisters in Wissenschaft und Praxis vom Lohnfertiger zum spezialisierten Geschäftspartner. Um die Expertise des CM-Dienstleisters effektiv zu nutzen, wird das Management von Wissen in Beziehungen zwischen OEMs und CM-Dienstleistern untersucht. Forschungsthemen sind insbesondere die bedarfsgerechte Bereitstellung und Erweiterung des Wissens im Rahmen der Zusammenarbeit (Yam et al. 2007; Gray et al. 2009). Komplementär beschäftigt sich die Forschung mit dem Kräfteverhältnis und der Dynamik der Geschäftsbeziehung (Gray et al. 2009; Denicolai et al. 2015; Ibrahim und Altahawi 2018). Infolge der Entwicklung von CM-Dienstleistern mit spezifischem Fachwissen und großer Markt- und Verhandlungsmacht nehmen diese zunehmend Einfluss auf die Steuerung der Geschäftsbeziehung und gestalten den Anwendungskontext von Management-Praktiken durch eigene Standards aktiv mit.

Die Schnittstelle zwischen Unternehmen und CM-Dienstleister gilt als kritisches Element für die Supply-Chain-Planung. Die Forschung beschäftigt sich in diesem Rahmen beispielsweise mit der Kapazitätsplanung sowie der Produktions- und Logistikplanung (Kim et al. 2002; Kaipia und Holmström 2007; Azadian et al. 2015). Zudem wird die Integration von Contract-Manufacturern in das Sales \& Operations Planning (Ivert und Jonsson 2010, 2014; Goh und Eldrigde 2015) intensiv erforscht. Diese Forschungsarbeiten beschränken sich auf die Integration der CM-Dienstleister in Prozesse und Systeme. Ausführungen zur zielgerichteten Steuerung und Kontrolle operativer Prozesse über die Integration hinaus finden sich hier nicht. Der Einfluss spezifischer Leistungsziele auf die Komplexität der Prozesssteuerung (Webster et al. 1997; Guzman 2003; Boulaksil und Fransoo 2010) ist eine Forschungsströmung, welche die Notwendigkeit der Betrachtung des Anwendungskontexts von Management-Praktiken stützt. Spezifische Management-Praktiken im Contract Manufacturing werden vereinzelt im Rahmen des interorganisationalen Qualitätsmanagements untersucht (Handley und Gray 2015; Sharma und Modgil 2015).

Kang et al. (2012) weisen jedoch die Relevanz einer übergreifenden organisatorischen Steuerung und Kontrolle der fremdvergebenen Fertigungsprozesse 
beim CM-Dienstleister nach. Zu diesem Zweck untersuchen sie, ob die Zielstellung der externen Fertigung Unternehmen bei der Anwendung von ManagementPraktiken für die Steuerung und Kontrolle beeinflusst (Kang et al. 2014).

\subsubsection{Forschungslücken und künftiger Forschungsbedarf}

Die Notwendigkeit eines ganzheitlichen Management-Ansatzes wird durch Kang et al. (2012) herausgestellt und gewinnt vor dem Hintergrund der dynamischen Entwicklung des Contract Manufacturing weiter an Bedeutung. Eine ganzheitliche Betrachtung von Anforderungen an das Management von CM-Dienstleistern und zugehörige Praktiken sind in der Literatur nicht zu finden. Webster et al. (1997) haben nachgewiesen, dass Unternehmen der Elektronikindustrie verschiedene Management-Ansätze gegenüber bestimmten CM-Dienstleistern verwenden. Kang et al. (2014) erweitern diese Betrachtung branchenübergreifend und belegen, dass die Ausprägung der Managementansätze mit Zielen der Fremdvergabe korrelieren, erfassen jedoch keine in der Praxis eingesetzten ManagementPraktiken. Daher besteht in der Forschung keine Transparenz darüber, welche Kontroll- und Steuerungspraktiken Unternehmen in diesem Zusammenhang nutzen. Folglich kann die Eignung der eingesetzten Management-Praktiken für die identifizierten situativen Management-Ansätze nicht nachvollzogen werden. Dies ist insbesondere relevant für das Verständnis, warum Fremdvergaben von Fertigungsprozessen scheitern und wie dem entgegengewirkt werden kann. Im Kontext des Contract Manufacturing besitzen verschiedene unternehmensinterne Organisationseinheiten durch ihre Schnittstellen einen Steuerungs- und Kontrollbedarf gegenüber dem CM-Dienstleister. Die organisatorische Verankerung und die Zuständigkeit für das Management von CM-Dienstleistern sind in diesem Kontext jedoch noch weitestgehend unerforscht.

Demnach stellen die Organisation und Durchführung der Steuerung und Kontrolle von CM-Dienstleistern mittels spezifischer Management-Praktiken durch Unternehmen eine bisher wenig betrachtete Forschungslücke dar, welche jedoch für den Erfolg von Fremdvergaben von Fertigungsprozessen von hoher Relevanz ist.

\subsection{Management von Contract-Manufacturing- Dienstleistern in der Praxis}

\subsubsection{Forschungsfragen und Denkrahmen}

Basierend auf dem Stand der Forschung und der praktischen Relevanz der Steuerung und Kontrolle von CM-Dienstleistern konzentriert sich dieser Beitrag auf ausgewählte Forschungsfragen, um den Rahmen zukünftiger Forschung 
ein- sowie abzugrenzen und ein tiefergehendes Verständnis für die praktischen Handlungsfelder des Managements von CM-Dienstleistern zu identifizieren. Zu diesem Zweck wird ein erster Denkrahmen für das Management von CM-Dienstleistern aufgestellt. Der Denkrahmen dient als Hilfestellung zur Strukturierung des Untersuchungsgegenstands anhand der wissenschaftlichen Forschungslücken und des in Studien nachgewiesenen Praxisbedarfs. Nachfolgend werden die drei Forschungsfragen in den Denkrahmen eingeordnet (siehe Abb. 8.1).

Kang et al. (2014) unterteilen Management-Praktiken in „Output Control“, „Process Control“" sowie „Social Control“" und adressieren damit das Ergebnis der Leistungserbringung, die Prozessdurchführung und die sozialen Komponenten der Zusammenarbeit. Kontrolle umfasst die Überwachung und Evaluation der Leistungserbringung nach definierten Kenngrößen. Durch Steuerungspraktiken wird ein CM-Dienstleister beeinflusst, die Leistungserbringung in einer vom Unternehmen beabsichtigten Weise durchzuführen. Aus dieser Grundstruktur lässt sich eine Typisierung (siehe Tab. 8.1) von Management-Praktiken ableiten.

Der Anwendungskontext von Management-Praktiken umfasst Elemente, welche die Eignung und Anwendung spezifischer Management-Praktiken beein-

\begin{tabular}{|l|}
\hline \multicolumn{1}{|c|}{ Forschungsstand } \\
\hline $\begin{array}{l}\text { Contract Manufacturing wird in der } \\
\text { Forschung vorrangig bis zum Zeitpunkt } \\
\text { der Fremdvergabe betrachtet und } \\
\text { untersucht. }\end{array}$ \\
$\begin{array}{l}\text { Das Management on CM-Dienstleistern } \\
\text { nach der Fremdvergabe wird in der } \\
\text { Literatur in ersten Ansätzen vereinzelt } \\
\text { als Aufgabe von Unternehmen } \\
\text { angesehen, welche als } \\
\text { organisatorische Einheit Management- } \\
\text { Praktiken abhängig von unbestimmten } \\
\text { Kontextelementen auswählen und } \\
\text { anwenden. }\end{array}$ \\
\hline
\end{tabular}

\begin{tabular}{ll|l|}
\multicolumn{2}{c|}{ Forschungsfragen } & \multicolumn{1}{c|}{ Praxisbedarf } \\
$\begin{array}{l}\text { Welche Kontextelemente beeinflussen die } \\
\text { situative Anwendung der Praktiken für das } \\
\text { Management von CM-Dienstleistern? } \\
\text { Welche Management-Praktiken setzen } \\
\begin{array}{l}\text { Unternehmen situativ ein, um ihre CM- } \\
\text { Dienstleister zu steuern und kontrollieren? } \\
\text { Wie koordinieren Unternehmen die } \\
\text { Anwendung der Management-Praktiken } \\
\text { zwischen den internen Stakeholdern? }\end{array}\end{array}$ \\
$\begin{array}{l}\text { unterliegt in der Praxis einer hohen } \\
\text { Intransparenz. } \\
\text { Spezifische Management-Praktiken } \\
\text { werden nicht gezielt identifiziert. } \\
\text { Unternehmen gestalten ihren } \\
\text { Management-Ansatz erfahrungsbasiert. } \\
\text { Die Kontextindividualität der externen } \\
\text { Fertigung erschwert den } \\
\text { unternehmensübergreifenden Vergleich } \\
\text { und Bewertung von Praktiken. } \\
\text { Die Zuständigkeit interner Stakeholder ist } \\
\text { häufig intransparent und erhöht die } \\
\text { Komplexität bei der Steuerung und } \\
\text { Kontrolle externer Fertigungsprozesse }\end{array}$ \\
\hline
\end{tabular}

Abb. 8.1 Einordnung der Forschungsfragen in den Denkrahmen

Tab. 8.1 Einordnung von Management-Praktiken im Contract Manufacturing (abgeleitet aus Kang et al. 2014)

\begin{tabular}{l|l|l}
\hline & Steuerung & Kontrolle \\
\hline Ergebnis & $\begin{array}{l}\text { (ES): Beeinflussung des CM-Dienst- } \\
\text { leisters hinsichtlich der Kenngrößen } \\
\text { der extern gefertigten Produkte }\end{array}$ & $\begin{array}{l}\text { (EK): Überprüfung der Einhaltung ver- } \\
\text { einbarter Kenngrößen in Bezug auf die } \\
\text { extern gefertigten Produkte }\end{array}$ \\
\hline Prozess & $\begin{array}{l}\text { (PS): Beeinflussung des CM-Dienst- } \\
\text { leisters hinsichtlich der Art und Weise } \\
\text { der Durchführung der Fertigungs- } \\
\text { prozesse }\end{array}$ & $\begin{array}{l}\text { (PK): Überprüfung der Einhaltung ver- } \\
\text { einbarter Kenngrößen in Bezug auf die } \\
\text { Durchführung der Fertigungsprozesse }\end{array}$ \\
\hline Sozial & $\begin{array}{l}\text { (SS): Beeinflussung des CM-Dienst- } \\
\text { leisters hinsichtlich der prozessüber- } \\
\text { greifenden Zusammenarbeit }\end{array}$ & $\begin{array}{l}\text { (SK): Überprüfung der Einhaltung ver- } \\
\text { einbarter Kenngrößen in Bezug auf die } \\
\text { prozessübergreifende Zusammenarbeit }\end{array}$ \\
\hline
\end{tabular}


flussen können. Nach Kang et al. (2014) können beispielsweise Leistungsziele wie Kosteneffizienz und Innovativität Elemente des Anwendungskontextes sein.

Zur Beantwortung der Forschungsfragen werden empirische Daten aus der pharmazeutischen Industrie erhoben. Contract Manufacturing umfasst in vielen Unternehmen der pharmazeutischen Industrie einen beachtlichen Umfang der fertigungsbezogenen Wertschöpfung (Singh et al. 2016). Pharmaunternehmen gehen gezielt Partnerschaften mit Contract-Manufacturern ein, um Zugang zu externem Know-how, Technologien und Märkten zu erhalten, Transaktionskosten zu senken und sich auf ihre eigenen Kernkompetenzen zu konzentrieren (Zhang et al. 2013). Das Management von CM-Dienstleistern bei der Herstellung pharmazeutischer Produkte wird aufgrund der technischen Komplexität des Fertigungsprozesses und regulatorischer Vorgaben als besonders komplex erachtet, gilt bisher jedoch als unzureichend dokumentiert und erforscht (Jakovcic 2008; Boulaksil und Fransoo 2010; Singh et al. 2016). Daher ist davon auszugehen, dass Unternehmen der Pharmabranche Expertise im Hinblick auf das Management von CM-Dienstleistern besitzen.

\subsubsection{Fallstudien für das Management von Contract- Manufacturing-Dienstleistern in der Praxis}

\subsubsection{Schema der Fallstudienanalysen}

Die Fallstudienerhebung folgt einem explorativen Ansatz, um aus dem Vergleich der Fälle Muster abzuleiten, welche zur Formulierung neuer Aussagen für die weitere Forschung beitragen. Da Geschäftsbeziehungen zu CM-Dienstleistern individuell und im Rahmen der Forschungsfragen schwer miteinander vergleichbar sind, wurden keine konkreten Anwendungsfälle betrachtet, sondern die Systematik des Managements von CM-Dienstleistern im Unternehmen erkundet. Die Fallstudien gliedern sich zur Beantwortung der Forschungsfragen inhaltlich in drei thematische Abschnitte (siehe Tab. 8.2).

Im ersten Teil der Fallstudienanalyse wurden Rahmenbedingungen für das Contract Manufacturing anhand des Unternehmensprofils, der Motivation für die Einbindung von CM-Dienstleistern, der Anzahl eingebundener CM-Dienstleister und des fremdvergebenen Fertigungsvolumens erfasst. Absolute Daten zur Höhe des fremdvergebenen Fertigungsvolumens und der Anzahl eingebundener CM-Dienstleister wurden für eine bessere fallübergreifende Vergleichbarkeit in eine Ordinalskala überführt. Das Skalierungsschema für die ordinal skalierten Merkmale und die Ausprägungen sind in Tab. 8.3 dargestellt.

Im dritten Teil wurde erhoben, welche Organisationseinheiten innerhalb eines betrachteten Unternehmens in das Management der CM-Dienstleister involviert sind. Es wurde weitergehend ermittelt, wer für die Koordination der Stakeholder und den Einsatz spezifischer Management-Praktiken zuständig ist. 
Tab. 8.2 Vorgehen bei der Fallstudienerhebung (abgeleitet aus Eisenhardt 1998)

\begin{tabular}{|c|c|}
\hline Schritt & Aktivität \\
\hline Initialisierung & $\begin{array}{l}\text { Wahl geeigneter Fallstudien und Instrumente für die Beantwortung der } \\
\text { Forschungsfragen: } \\
\text { Welche Kontextelemente beeinflussen die situative Anwendung der Praktiken } \\
\text { für das Management von CM-Dienstleistern? } \\
\text { Welche Management-Praktiken setzen Unternehmen situativ ein, um ihre CM- } \\
\text { Dienstleister zu steuern und zu kontrollieren? } \\
\text { Wie koordinieren Unternehmen die Anwendung der Management-Praktiken } \\
\text { zwischen den internen Stakeholdern? }\end{array}$ \\
\hline Fallstudien & $\begin{array}{l}\text { Sechs Unternehmensfallstudien als Querschnitt der Schweizer Pharmabranche } \\
\text { hinsichtlich Unternehmensgröße und Kernkompetenzen }\end{array}$ \\
\hline Instrumente & $\begin{array}{l}\text { Durchführung: } \\
\text { Zehn semi-strukturierte Interviews mit FachexpertInnen für die Anwendung } \\
\text { von Praktiken zum Management von Contract Manufacturern im jeweiligen } \\
\text { Unternehmen. Die FachexpertInnen zeichnen sich durch mehrjährige Praxis- } \\
\text { erfahrung basierend auf einer (Teil-) Verantwortung der Steuerung und } \\
\text { Kontrolle von CM-Dienstleistern im jeweiligen Unternehmen aus. In Fall- } \\
\text { studie } 1 \text { wurden aufgrund der aufgabenbezogenen, dezentralen Zuständigkeit } \\
\text { fünf Interviews durchgeführt. In den übrigen Fallstudien wurde jeweils ein } \\
\text { Ansprechpartner pro Unternehmen befragt. Den FachexpertInnen wurden } \\
\text { die Interviews zur internen Vorbereitung vorab vorgestellt. Der Frage- } \\
\text { bogen des Interviews war für alle Interviewpartner konsistent. Im Rahmen } \\
\text { des semi-strukturierten Interview-Ansatzes wurde den Befragten die offene } \\
\text { Beantwortung der Fragen inklusive Nachfragen eingeräumt. Die Interviews } \\
\text { hatten einen Zeitumfang von } 60 \text { min und wurden von zwei wissenschaftlichen } \\
\text { Mitarbeitenden durchgeführt. Die Audiospur der Interviews wurde auf- } \\
\text { genommen und transkribiert } \\
\text { Auswertung: } \\
\text { Die Auswertung erfolgte auf Basis der transkribierten Audiospur des Interviews } \\
\text { nach dem 4-Augen-Prinzip durch zwei wissenschaftliche Mitarbeitende }\end{array}$ \\
\hline Datenanalyse & $\begin{array}{l}\text { Schritt 1: Jeweils innerhalb der Fallstudien } \\
\text { Schritt 2: Fallstudienübergreifend }\end{array}$ \\
\hline $\begin{array}{l}\text { Beitrag zur } \\
\text { Forschung }\end{array}$ & $\begin{array}{l}\text { Die Erhebung schafft neue Erkenntnisse bezüglich in der Praxis verbreiteter } \\
\text { Praktiken und Anwendungskontexte zum Management von CM-Dienstleistern. } \\
\text { Konkret untersucht wurden: } \\
\text { Kontextelemente, welche die Anwendung spezifischer Management-Praktiken } \\
\text { beeinflussen } \\
\text { in der Praxis angewendete Praktiken zum Management von CM-Dienstleistern } \\
\text { die organisationsinterne Zuständigkeit für die Koordination der Stakeholder } \\
\text { und die Anwendung von Management-Praktiken }\end{array}$ \\
\hline
\end{tabular}

\subsubsection{Fallstudie 1}

Gegenstand ist ein globales Pharmaunternehmen, das eigene Pharmaprodukte erforscht, entwickelt, herstellt sowie vertreibt und sich selbst als Partner für innovative patientenorientierte Lösungen sieht. Die Produktbereiche des Unternehmens unterteilen sich in Eisenmangel-Therapien und Mittel gegen Infektionskrankheiten. 
Tab. 8.3 Ordinales Skalierungsschema der Anzahl eingebundener CM-Dienstleister und fremdvergebenen Fertigungsvolumens

\begin{tabular}{l|l|l|l}
\hline & Niedrig & Mittel & Hoch \\
\hline Anzahl ein- & Der Quotient aus & Der Quotient aus & Der Quotient aus \\
gebundener & Umsatz und Anzahl & $\begin{array}{l}\text { Umsatz und Anzahl } \\
\text { eingebundener CM- }\end{array}$ & $\begin{array}{l}\text { Umsatz und Anzahl } \\
\text { eingebundener CM- }\end{array}$ \\
$\begin{array}{l}\text { eingebundener CM- } \\
\text { Dienstleister }\end{array}$ & Dienstleister ist $<5$ & Dienstleister ist 5-15 & Dienstleister ist $>15$ \\
\hline Fremdver- & Der Anteil des fremd- & Der Anteil des fremd- & Der Anteil des fremd- \\
gebenes & vergebenen Fertigungs- & vergebenen Fertigungs- & vergebenen Fertigungs- \\
Fertigungs- & volumens beträgt<30\% & volumens beträgt & volumens beträgt $>70 \%$ \\
volumen & des gesamten & $30-70 \%$ des gesamten & des gesamten Fertigungs- \\
& Fertigungsvolumens & Fertigungsvolumens & volumens \\
\hline
\end{tabular}

Grundsätzlich bevorzugt das Unternehmen eine interne Fertigung der eigenen Produkte, ist aber auf den Zugang zu spezifischen Fertigungstechnologien und externen Kapazitäten angewiesen. Externe Contract Manufacturer werden in einem Dual-Source-Ansatz komplementär zu internen Fertigungskapazitäten eingesetzt. Der Contract-Manufacturing-Anteil der Fertigung von über $60 \%$ bewegt sich, wie die Anzahl eingesetzter CM-Dienstleister im mittleren Bereich. Aufgrund hoher Wechselbarrieren beim Zugang zu bestimmten Technologien und Märkten ist das Unternehmen von seinen CM-Dienstleistern in hohem Maße abhängig. Die aktuellen Geschäftsbeziehungen des Unternehmens zu CM-Dienstleistern lassen sich als transaktional einstufen. Eine engere Zusammenarbeit wird nicht angestrebt.

Der Fokus der Steuerung und Kontrolle von CM-Dienstleistern liegt für das Unternehmen in der Gewährleistung der zeit- und qualitätsgerechten Lieferleistung. Zu diesem Zweck werden definierte Kennzahlen erhoben und überwacht. Die Kennzahlen sind an ein informelles Anreizsystem gebunden, durch welches die leistungsstärksten CM-Dienstleister bei der Vergabe von Fertigungsaufträgen intern bevorzugt werden. Ergänzt wird die Ergebniskontrolle durch eine Prozesskontrolle in Form von Qualitäts-Audits in Abstimmung mit behördlichen Vorschriften. In Abb. 8.2 sind die erfassten Management-Praktiken dargestellt.

Die Zuständigkeit für die Auswahl und Anwendung von ManagementPraktiken ist im Unternehmen dezentral auf die verschiedenen Fachabteilungen verteilt. Diese sind aufgabenbezogen für Teilaufgaben beim Management des CM-Dienstleisters, wie der Kontrolle einzelner Kennzahlen, zuständig. Welche Fachabteilung zu welchem Zeitpunkt mit dem CM-Dienstleister in Kontakt steht, ist vom Lebenszyklus des zu fertigenden Produkts abhängig. Erster Ansprechpartner zu grundlegenden oder fachübergreifenden Anfragen ist die Supply-ChainManagement-Abteilung.

\subsubsection{Fallstudie 2}

Die zweite Fallstudie betrifft einen globalen Marktführer auf dem Gebiet von Lungenerkrankungen. Weitere Tätigkeitsgebiete des Unternehmens umfassen 


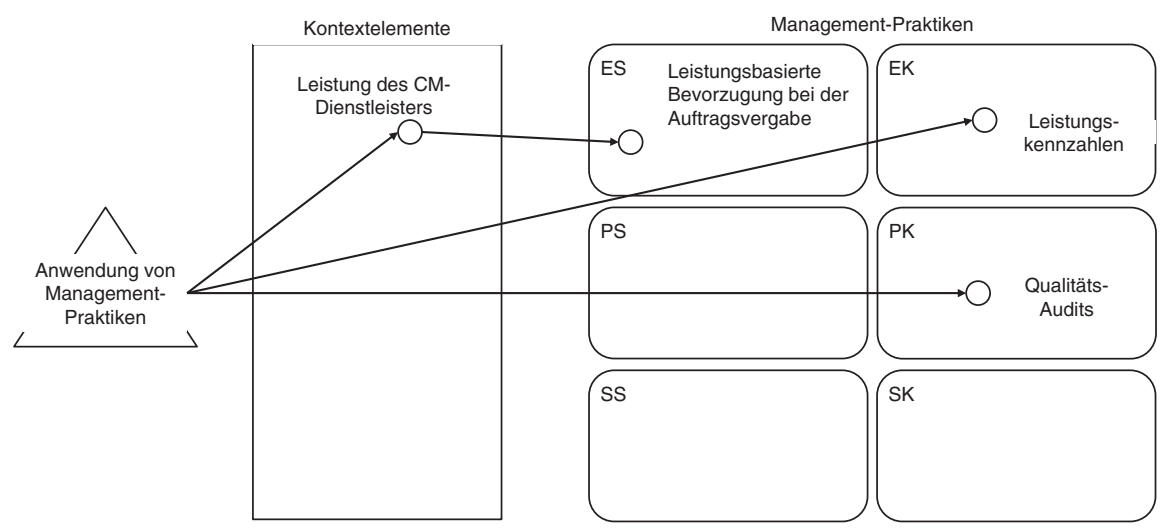

Abb. 8.2 In Fallstudie 1 erfasste Management-Praktiken und ihr Anwendungskontext

die Bereiche Herz-Kreislauferkrankungen, Infektionskrankheiten, Neurowissenschaften und die Onkologie.

Das Unternehmen sieht seine Kernkompetenzen in der Entwicklung sowie Distribution von Medikamenten und verfolgt eine klare Fremdfertigungsstrategie. Lediglich Teile der Wirkstoffentwicklung werden intern durchgeführt. Das hohe Fremdfertigungsvolumen wird von einem mittelgroßen Pool von CM-Dienstleistern erbracht. Die Zusammenarbeit mit Contract-Manufacturern wird vom Unternehmen als rein transaktionale Geschäftsbeziehung verstanden. Lediglich mit wenigen, finanziell bedeutenden Geschäftspartnern werden in Jahresgesprächen strategische Verbesserungspotenziale avisiert.

Von hoher Relevanz unter den Management-Praktiken ist für das Unternehmen die Kontrolle der vereinbarten Leistungsziele. Zu diesem Zweck werden Leistungskennzahlen definiert, welche an ein finanzielles Anreizsystem angebunden sind, um eigenständige Kennzahlverbesserungen $\mathrm{zu}$ incentivieren. In Jahresgesprächen mit strategisch wichtigen CM-Dienstleistern finden gegenseitige Bewertungen statt, auf deren Basis Verbesserungspotenziale erarbeitet werden. Auf Prozessebene werden Qualitäts-Audits durchgeführt, um potenzielle Qualitätsrisiken frühzeitig aufzudecken und zu beheben. Im Falle von Störfällen behält sich das Unternehmen vor, kooperativ an der Fehlerbehebung mitzuwirken. Abb. 8.3 zeigt die erfassten Management-Praktiken.

Die Zuständigkeit für die Durchführung der Management-Praktiken obliegt dem technischen Projektmanagement. Dessen Hauptaufgabe ist die Konfiguration von Fertigungslinien u. a. beim CM-Dienstleister. Im weiteren Verlauf der Geschäftsbeziehung bleibt das technische Projektmanagement zentraler Ansprechpartner für das Management der CM-Dienstleister. Account Manager innerhalb der Organisationseinheit werden spezifischen CM-Dienstleistern als Kontaktpersonen zugewiesen. Die Account Manager koordinieren die Anwendung von Management-Praktiken in Abstimmung mit beteiligten Fachabteilungen. 


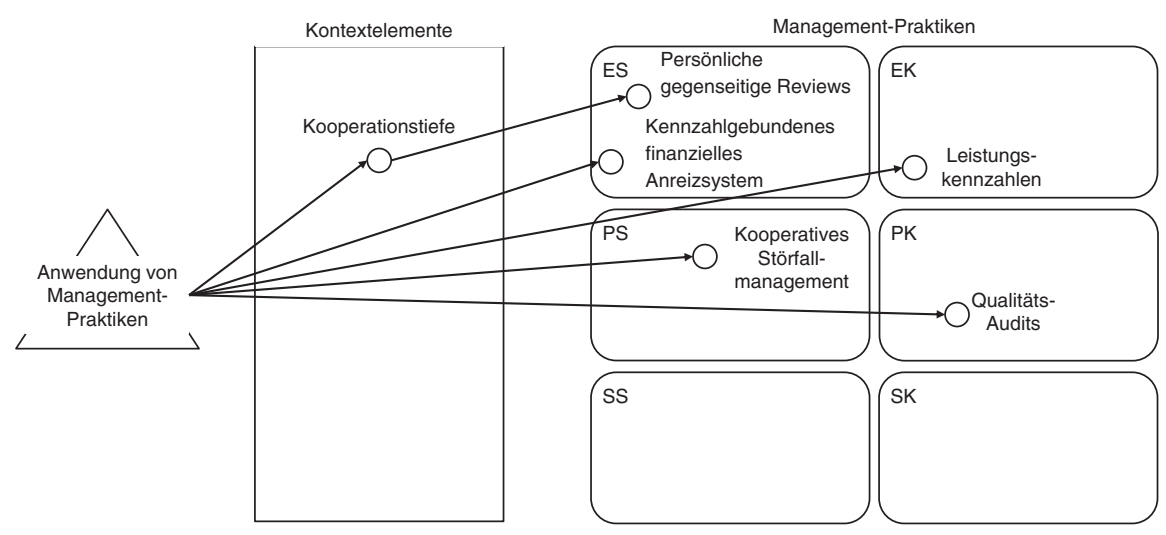

Abb. 8.3 In Fallstudie 2 erfasste Management-Praktiken und ihr Anwendungskontext

\subsubsection{Fallstudie 3}

Gegenstand der dritten Fallstudie ist ein global aktives Pharmaunternehmen, welches ein breit diversifiziertes Produkt- und Technologieportfolio aufweist. Das Unternehmen bietet sowohl generische Medikamente als auch Produkte für die Diagnostik und Therapie auf Gebieten wie der Onkologie, Virologie, Neurologie und Transplantationsmedizin an. Darüber hinaus ist es im Bereich der Biotechnologie aktiv und stellt neben Medikamenten ebenfalls medizinische Geräte her.

Aufgrund der Bandbreite an Aktivitäten lassen sich die Hauptziele für die Einbindung von CM-Dienstleistern nicht übergreifend zusammenfassen. Sie unterscheiden sich von Produktgruppe zu Produktgruppe stark und hängen von spezifischen Faktoren, wie dem eigenen Know-how, Technologien sowie vom Produktlebenszyklus ab. Das Fremdfertigungsvolumen ist über alle Produktgruppen hinweg als mittelgroß zu bewerten. Die Anzahl eingebundener CM-Dienstleister ist gering und wird aktiv konsolidiert. Der Einsatz von Management-Praktiken wird durch das unternehmerische Risiko der Partnerschaft bestimmt. Die CM-Dienstleister werden basierend auf dem vergebenen Fertigungsvolumen, der Leistungshistorie des Dienstleisters und der technischen Komplexität des Fertigungsprozesses in Risikosegmente eingeordnet. Innerhalb dieser Risikosegmente werden spezifische Praktiken angewendet.

Das Unternehmen kontrolliert die Leistung seiner CM-Dienstleister anhand definierter Leistungskenngrößen. Kennzahlgebundene, vertraglich vereinbarte Bonus- und Strafzahlungen werden eingesetzt, um eine selbstständige Leistungsverbesserung anzuregen. Neben Qualitäts-Audits werden weitere „Safety, Health \& Environment" (SHE)-Audits durchgeführt. Diese dienen dazu, die Einhaltung von Compliance-Vereinbarungen durchzusetzen. Zudem treibt das Unternehmen die Digitalisierung der Informationsprozesse voran. CM-Dienstleister werden abhängig vom Business Case entweder direkt mit dem ERP des Unternehmens 


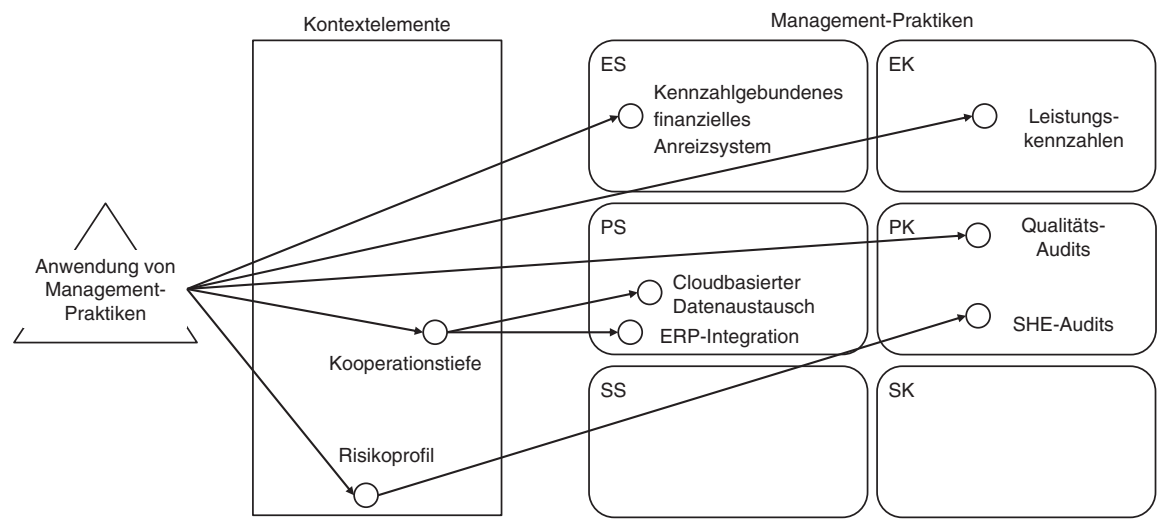

Abb. 8.4 In Fallstudie 3 erfasste Management-Praktiken und ihr Anwendungskontext

verbunden oder dazu angehalten, cloudbasierte Kooperations-Anwendungen zu nutzen. Abb. 8.4 gibt einen Überblick über die erfassten Management-Praktiken.

Die Zuständigkeit für die Steuerung und Kontrolle von CM-Dienstleistern ist im Unternehmen in einem hybriden Ansatz aus zentralen und dezentralen Aufgabenbereichen aufgeteilt. Eine primär für Contract Manufacturing zuständige, übergreifende Plattform initiiert und steuert strategische Projekte zur Verbesserung des Managements der CM-Dienstleister. Die Zuständigkeit für die operative Betreuung der CM-Dienstleister im Tagesgeschäft liegt bei dezentral organisierten Account Managern, welche der zentralen Plattform formal untergeordnet sind. Diese leiten interdisziplinäre Teams aus FachexpertInnen, um die ihnen zugeteilten CM-Dienstleister mit Management-Praktiken zu steuern und zu kontrollieren.

\subsubsection{Fallstudie 4}

Die vierte Fallstudie beschreibt einen diversifizierten und global aktiven Pharmakonzern, dessen Kernkompetenzen in der Entwicklung medizinischer Wirkstoffe liegen. Das Unternehmen ist u. a. in der Onkologie, bei Impfstoffen und Diagnostika, in der Augenheilkunde und bei Generika aktiv.

Das Unternehmen verfolgt die Strategie, innovative Produkte und Technologien im Unternehmen zu konzentrieren. Es wird ein hohes Fremdfertigungsvolumen inklusive der kommerziellen Produktfertigung extern an CM-Dienstleister vergeben. Um Management-Ressourcen bedarfsgerecht einzusetzen, unterscheidet das Unternehmen vier verschiedenen Gruppen von CM-Dienstleistern. Leistungsstarke CM-Dienstleister werden gezielt gefördert und enger eingebunden. Die zahlenmäßig größte Gruppe bilden CM-Dienstleister für Generika, welche aktiv konsolidiert werden. Diese Gruppe wird nicht aktiv gesteuert und vorranging nach Kostenführerschaft beurteilt. Zusätzlich existieren zwei weitere Segmente 
von CM-Dienstleistern, welche dem Unternehmen den Zugang zu spezifischen Technologien und Märkten eröffnen. Hier strebt das Unternehmen eine möglichst stabile Geschäftsbeziehung an.

Die Leistungskontrolle der CM-Dienstleister anhand spezifischer Kennzahlen stellt für das Unternehmen eine zentrale Management-Praktik dar. Vertraglich verankerte Bonuszahlungen dienen den CM-Dienstleistern als Incentivierung zur Leistungsverbesserung. Mit der Durchführung von Qualitäts-Audits wird zudem die ordnungsgemäße Prozessdurchführung durch den CM-Dienstleister überprüft. Cloudbasierte Kommunikationssysteme werden eingesetzt, um den Datenaustausch zu digitalisieren und zu automatisieren. Präferierte CM-Dienstleister werden direkt an das ERP-System des Unternehmens angebunden, erhalten Einsicht in Prognosedaten und werden durch Qualifizierungsmaßnahmen gezielt unterstützt sowie entwickelt. Schulungen und Workshops sollen die Zusammenarbeit und Leistungsfähigkeit des CM-Dienstleisters stärken. Audit-Simulationen dienen der Prüfungsvorbereitung. In Abb. 8.5 sind die erfassten ManagementPraktiken dargestellt.

Die Zuständigkeit für die Anwendung der Management-Praktiken ist im Unternehmen dezentral verteilt. Innerhalb der Organisation „Technisches Projektmanagement" werden Account Manager eingesetzt, welche in spezifischen Produkt- oder Ländergruppen aktiv sind und die Schnittstelle zum CM-Dienstleister darstellen. Die Account Manager sind für die Steuerung und Kontrolle der ihnen zugewiesenen CM-Dienstleister zuständig und werden durch AnwendungsexpertInnen aus unternehmensinternen Fachabteilungen unterstützt.

\subsubsection{Fallstudie 5}

Gegenstand der fünften Fallstudie ist ein global agierendes Pharmaunternehmen, welches zu den führenden Biotechnologie-Anbietern und -Anwendern gehört. Das

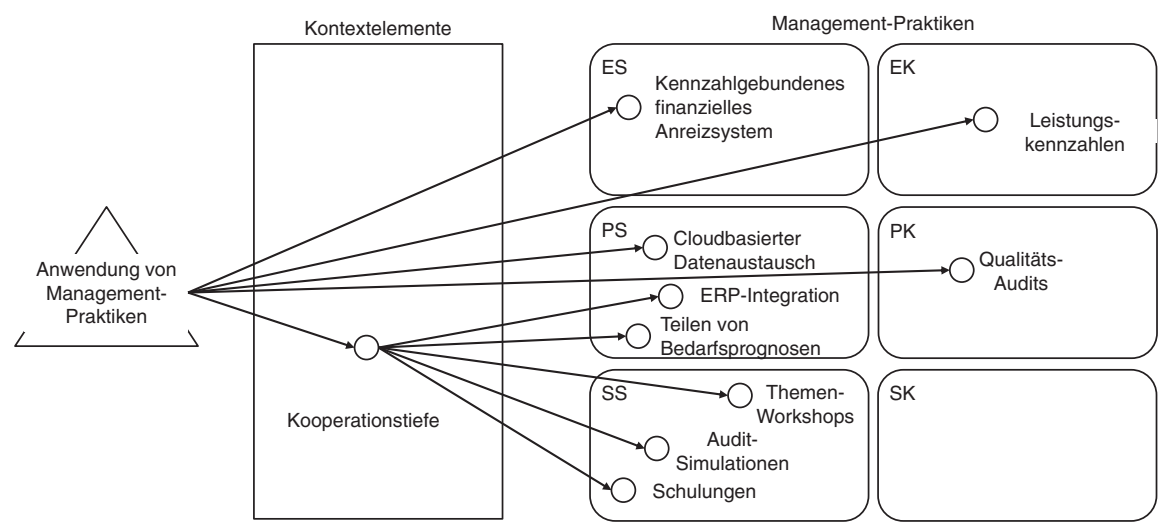

Abb. 8.5 In Fallstudie 4 erfasste Management-Praktiken und ihr Anwendungskontext 
Unternehmen forscht und entwickelt Medikamente auf den Gebieten der Neurologie, Immunologie und Hämatologie.

Biotechnologische Verfahren werden im Unternehmen als Kernkompetenz angesehen und durch eine klare Fremdvergabestrategie der Fertigung unterstützt. Insbesondere für die kommerzielle Fertigung greift das Unternehmen auf CM-Dienstleister zurück. Das Fremdfertigungsvolumen des Unternehmens ist hoch die Anzahl eingesetzter CM-Dienstleistern jedoch gering. Diese werden in risikobasierte Management-Segmente eingeteilt, welche die Grundlage für die Anwendung spezifischer Praktiken bilden. Die Segmente werden auf Basis der Entwicklung von Risikofaktoren und Leistungskennzahlen dynamisch aktualisiert, wodurch sich eine fortlaufende Anpassung des Management-Ansatzes gegenüber einzelnen CM-Dienstleistern ergibt.

Zur bedarfsgerechten Anpassung der Management-Segmente, stellt die kennzahlbasierte Leistungskontrolle für das Unternehmen eine zentrale Praktik dar. Durch die Integration der IT-Systeme sowie den Einsatz spezifischer Software werden echtzeitnahe Kennzahlenreports generiert. Die Kennzahlenreports werden bei Bedarf wöchentlich mit den CM-Dienstleister besprochen. Befinden sich einzelne Kenngrößen eines CM-Dienstleisters über einen längeren Zeitraum in einem nicht wünschenswerten Bereich, werden kennzahlenspezifische Audits zur Ursachenanalyse durchgeführt. Diese ergänzen regulär durchgeführte QualitätsAudits. Abb. 8.6 visualisiert die erfassten Management-Praktiken.

Die Zuständigkeit für das Management der CM-Dienstleister ist organisatorisch in einem hybriden Ansatz in strategische und operative Tätigkeiten aufgeteilt. Für die Durchführung strategischer Aufgaben und die Koordination der internen Stakeholder ist eine zentrale Organisationseinheit zuständig. Dieser sind „virtuellen Werksleitern“ untergeordnet, welche CM-Dienstleister durch die Management-Praktiken im Tagesgeschäft operativ kontrollieren und steuern. In

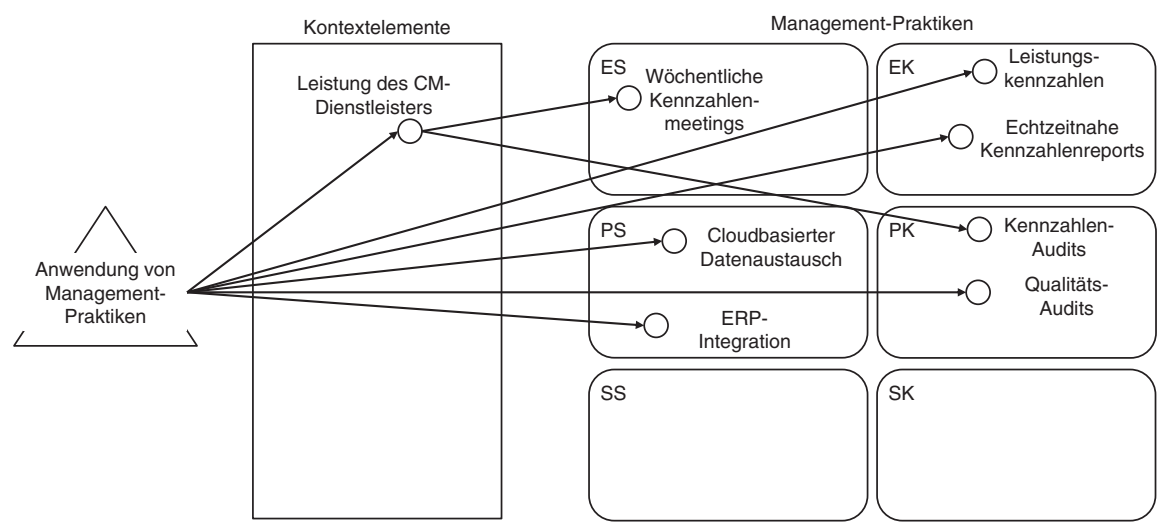

Abb. 8.6 In Fallstudie 5 erfasste Management-Praktiken und ihr Anwendungskontext 
ihrer Tätigkeit werden sie von dezentralen funktionsübergreifenden Teams mit fest zugeordneten ExpertInnen aus relevanten Fachabteilungen unterstützt.

\subsubsection{Fallstudie 6}

Gegenstand der sechsten Fallstudie ist ein weltweit im Bereich Life Science tätiges Unternehmen mit Produktionsstandorten in Europa, Nordamerika und Asien. Das Unternehmen entwickelt und produziert pharmazeutische Wirkstoffe und Zwischenprodukte für die forschende Pharmaindustrie.

Das Unternehmen ist dementsprechend selbst als CM-Dienstleister für Pharmaunternehmen tätig, vergibt jedoch ebenfalls Fertigungsaufträge an CM-Dienstleister. Gemessen an der Unternehmensgröße sind der Fremdfertigungsanteil und die Anzahl eingebundener CM-Dienstleister gering. Mit der Fremdfertigung beabsichtigt das Unternehmen, sein eigenes Leistungsportfolio gegenüber den KundInnen durch den Zugriff auf komplementäre Technologien zu ergänzen.

Das Unternehmen nutzt für die Leistungsbewertung der CM-Dienstleister quantitative und qualitative Leistungskennzahlen, welche in einem Punktesystem verrechnet werden. Zur Incentivierung der CM-Dienstleister werden alle Leistungskennzahlen konkurrierenden Anbietern anonym zugänglich gemacht. Als weiterer Anreiz kommen vertraglich fixierte Bonus- und Strafzahlungen zum Einsatz. Zur regelmäßigen Kontrolle der Fertigungsprozesse werden Qualitäts-Audits durchgeführt. Cloudbasierte Kommunikationssysteme werden eingesetzt, um den Kommunikationsprozess durch automatisierten Datenaustausch zu steuern. Abb. 8.7 zeigt die erfassten Management-Praktiken.

Die Zuständigkeit für das Management der CM-Dienstleister ist in die Organisationseinheit „Technisches Projektmanagement“ integriert. Diese ist in

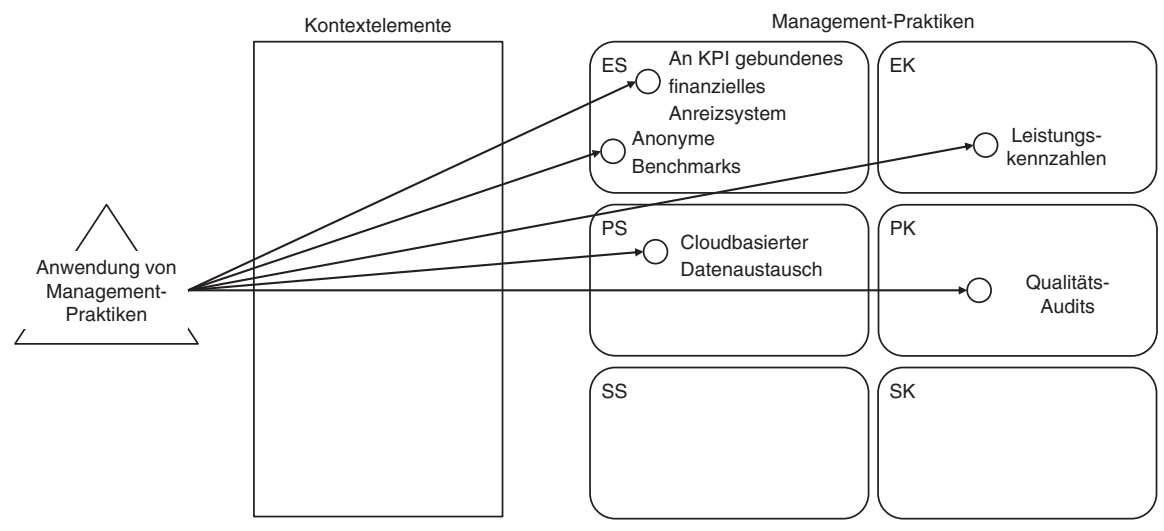

Abb. 8.7 In Fallstudie 6 erfasste Management-Praktiken und ihr Anwendungskontext 
ihrer Hauptfunktion für die Errichtung von Versuchs- und Fertigungsanlagen zuständig. Zusätzlich koordiniert und verantwortet sie alle Aktivitäten im Rahmen der Kontrolle und Steuerung der CM-Dienstleister.

\subsubsection{Cross-Case-Analyse der Fallstudien}

\subsubsection{Kontext der Fremdvergabe an CM-Dienstleister}

Die Fallstudien zeigen auf, dass der Anwendungskontext, in dem Pharma-Unternehmen Praktiken zur Steuerung und Kontrolle ihrer CM-Dienstleister einsetzen, für die Unternehmen selbst nicht immer transparent ist. Eine systematische Einordnung von CM-Dienstleistern in verschiedene Management-Segmente erfolgt lediglich bei der Hälfte der untersuchten Unternehmen.

Die Einteilung in unternehmensindividuelle Management-Segmente berücksichtigt Kontextelemente wie das technische und kommerzielle Risikoprofil, die Ziele der Fremdvergabe, die Leistungshistorie oder die Tiefe der Kooperation. Auch der Produktlebenszyklus wird explizit genannt.

Neben diesen explizit angeführten Kontextelementen sind implizit aus den Aussagen und Praktiken der betrachteten Unternehmen Hinweise auf weitere, zu berücksichtigende Größen zu entnehmen. Der auf den Zugang zu bestimmten Märkten ausgerichtete Einsatz von CM-Dienstleistern lässt darauf schließen, dass länderspezifische Regularien den Bedarf nach Steuerung und Kontrolle der CM-Dienstleister beeinflussen. Zudem erfahren neue Kooperationspartner eine strengere Kontrolle als etablierte CM-Dienstleister, wodurch die Dauer der Kooperation als ein relevantes Kontextelement interpretiert werden kann. Durch Kooperationen zum Zugang zu spezifischen Märkten und Technologien wird die Abhängigkeit von CM-Dienstleistern deutlich. CM-Dienstleister mit einer hohen Verhandlungsmacht können den Einsatz bestimmter Management-Praktiken ablehnen sowie die Steuerungs- und Kontrollaktivitäten der Unternehmen eingrenzen. Die befragten Unternehmen gaben ferner an, ihren Pool von CM-Dienstleistern aktiv verkleinern zu wollen, um verfügbare Management-Kapazitäten für die Vertiefung der Zusammenarbeit mit den verbleibenden Dienstleistern zu einzusetzen. Demnach spielen die Netzwerkgröße und die zur Verfügung stehenden Management-Kapazitäten eine große Rolle. Tab. 8.4 gibt einen Überblick über die identifizierten Elemente des Anwendungskontexts.

$\mathrm{Da}$ lediglich in drei Fallstudien eine kontextspezifische Auswahl von Management-Praktiken stattfindet, ist davon auszugehen, dass insbesondere die implizit identifizierten Elemente nicht vollständig sind. Dennoch ist festzuhalten, dass sich die Hälfte der untersuchten Pharmaunternehmen der Relevanz des Anwendungskontextes bewusst ist. Sie nutzen diesen explizit, um die Anwendung von Management-Praktiken bedarfsgerecht zu steuern. 
Tab. 8.4 Identifizierte Elemente des Anwendungskontextes

\begin{tabular}{l|l}
\hline $\begin{array}{l}\text { Explizit genannte Elemente des } \\
\text { Anwendungskontextes }\end{array}$ & $\begin{array}{l}\text { Abgeleitete implizite Elemente des } \\
\text { Anwendungskontextes }\end{array}$ \\
\hline Kommerzielles Risiko & Kräfteverhältnis/Verhandlungsmacht \\
\hline Technisches Risiko & Kooperationsdauer \\
\hline Fremdvergabeziele & Management-Kapazität \\
\hline Kooperationstiefe & Länderspezifische Regularien \\
\hline Produktlebenszyklus & \\
\hline Leistungshistorie & \\
\hline
\end{tabular}

\subsubsection{Kontextspezifische Anwendung von Management-Praktiken}

Unter den erfassten Management-Praktiken spielen die Ergebnissteuerung und -kontrolle eine dominante Rolle. Vier der sechs betrachteten Unternehmen nutzen kontextunabhängig Praktiken zur Incentivierung der CM-Dienstleister. Die konkrete Ausgestaltung der Incentivierungsmechanismen unterscheidet sich fallstudienübergreifend voneinander, ist jedoch grundsätzlich vertraglich fixiert und an zentrale Leistungskennzahlen gebunden. Zusätzlich wurden Benchmarks, basierend auf anonymisierten Kennzahlen-Reports, sowie eine Bevorzugung bei Auftragsvergaben als nicht-monetäre Incentivierungspraktiken identifiziert. Die Ergebniskontrolle erfolgt über definierte Leistungskennzahlen und ist durchgängig verbreitet.

Qualitäts-Audits sind aufgrund regulatorischer Anforderungen der Pharmabranche Standard beim Management von CM-Dienstleistern und werden in allen Fallstudien durchgeführt. Zusätzlich werden abhängig vom Risikoprofil oder der Leistung des Contract-Manufacturers Audits zu weiteren Kenngrößen eingesetzt. In die Steuerung der fremdvergebenen Fertigungsprozesse greifen die Unternehmen jedoch außerhalb des Störfallmanagements nicht ein. Informationsprozesse werden durch die Vorgabe von Kommunikationskanälen hingegen zunehmend aktiv gesteuert. Die Digitalisierung führt dazu, dass Unternehmen dazu übergehen, spezifische Kommunikationspraktiken durchzusetzen. Dies betrifft die Integration von IT-Systemen oder die Nutzung von cloudbasierten Plattformen, um den Datenaustausch abzuwickeln. Je nach Unternehmen ist dies bereits Standard oder wird kontextspezifisch mit relevanten Partnern durchgeführt.

Management-Praktiken zur gezielten Entwicklung der Geschäftsbeziehung konnten in Fallstudie 4 identifiziert werden. Management-Praktiken im Bereich der sozialen Steuerung sind Qualifikationsmaßnahmen im weiteren Sinne. Soziale Management-Praktiken werden im Lichte verbundenen, hohen internen Aufwands exklusiv bei strategischen Partnern eingesetzt.

Tab. 8.5 enthält die in den Fallstudien identifizierten Praktiken inklusive ihrer Gestaltungsvarianten und des identifizierten Anwendungskontexts. 
Tab. 8.5 Fallstudienübergreifende Management-Praktiken

\begin{tabular}{|c|c|c|c|}
\hline Kategorie & $\begin{array}{l}\text { Management- } \\
\text { Praktiken }\end{array}$ & $\begin{array}{l}\text { Elemente des } \\
\text { Anwendungskontexts }\end{array}$ & Gestaltungsvarianten \\
\hline \multirow{3}{*}{$\begin{array}{l}\text { Ergebnis- } \\
\text { steuerung } \\
\text { und -kontrolle }\end{array}$} & $\begin{array}{l}\text { Leistungskenn- } \\
\text { zahlen }\end{array}$ & & $\begin{array}{l}\text { Echtzeitnahe Kennzahlen- } \\
\text { Reports }\end{array}$ \\
\hline & $\begin{array}{l}\text { Incentivierungs- } \\
\text { praktiken }\end{array}$ & & $\begin{array}{l}\text { KPI-gebundene finanzielles } \\
\text { Anreizsysteme } \\
\text { Anonymisierte Benchmarks } \\
\text { Leistungsbasierte Bevorzugung } \\
\text { bei der Auftragsvergabe }\end{array}$ \\
\hline & $\begin{array}{l}\text { Evaluations- } \\
\text { praktiken }\end{array}$ & $\begin{array}{l}\text { Risikoprofil } \\
\text { Kooperationstiefe } \\
\text { Produktlebenszyklus }\end{array}$ & $\begin{array}{l}\text { Wöchentliche Kennzahlen- } \\
\text { meetings } \\
\text { Gegenseitige Reviews }\end{array}$ \\
\hline \multirow[t]{3}{*}{$\begin{array}{l}\text { Prozess- } \\
\text { steuerung } \\
\text { und -kontrolle }\end{array}$} & Audits & Leistungshistorie & $\begin{array}{l}\text { Qualitäts-Audits } \\
\text { Kennzahlen-Audits } \\
\text { SHE-Audits }\end{array}$ \\
\hline & \begin{tabular}{|l} 
Digitale \\
Kommunikations- \\
praktiken
\end{tabular} & Kooperationstiefe & $\begin{array}{l}\text { ERP-Integration } \\
\text { Cloudbasierter Datenaustausch } \\
\text { Teilen von Bedarfsprognosen }\end{array}$ \\
\hline & \begin{tabular}{|l|}
$\begin{array}{l}\text { Störfall- } \\
\text { management }\end{array}$ \\
\end{tabular} & - & $\begin{array}{l}\text { Kooperatives Störfall- } \\
\text { management }\end{array}$ \\
\hline $\begin{array}{l}\text { Sozialsteuerung } \\
\text { und -kontrolle }\end{array}$ & $\begin{array}{l}\text { Qualifikations- } \\
\text { maßnahmen }\end{array}$ & $\begin{array}{l}\text { Kooperationstiefe } \\
\text { Management-Kapazi- } \\
\text { täten }\end{array}$ & $\begin{array}{l}\text { Schulungen } \\
\text { Themen-Workshops } \\
\text { Audit-Simulationen }\end{array}$ \\
\hline
\end{tabular}

\subsubsection{Interne Koordination und Zuständigkeit für die Anwendung der Management-Praktiken}

Die Koordination der internen Stakeholder ist mit der organisatorischen Zuständigkeit für das Management der Dienstleister verknüpft. In diesem Zusammenhang wurden drei organisatorische Gestaltungsansätze identifiziert (Abb. 8.8): Zentrale oder dezentrale Zuständigkeit für das Management der CM-Dienstleister sowie einen hybriden Ansatz mit einer gezielten Trennung von Zuständigkeiten.
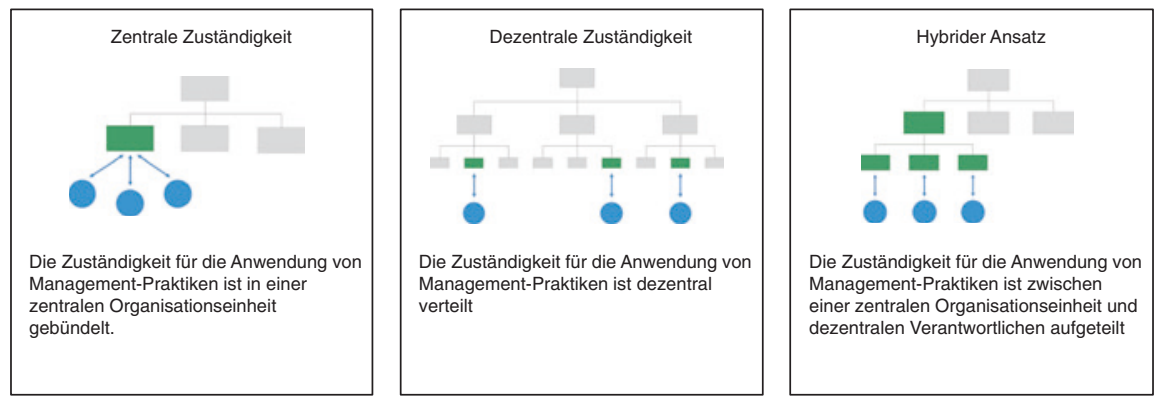

Abb. 8.8 In den Fallstudien erfasste Zuständigkeitsvarianten für die Steuerung und Kontrolle von Contract-Manufacturern 
In den Fallstudien 2 und 6 liegt die Zuständigkeit für das Management der CM-Dienstleister zentral in einer Organisationseinheit. Diese tritt dem Dienstleister als Ansprechpartner gegenüber und ist intern für die Koordination und Anwendung der Management-Praktiken zuständig. In beiden Fallstudien handelt es sich um keine spezialisierte Organisationseinheit, welche abseits vom Contract Manufacturing zusätzliche Aufgabenbereiche verantwortet. Eine Gemeinsamkeit der beiden Fallstudien liegt darin, dass beide Unternehmen nach eigenen Angaben mit einer geringen Anzahl an CM-Dienstleistern zusammenarbeiten.

In den Fallstudien 1 und 4 wird ein dezentraler Ansatz zur Steuerung und Kontrolle der CM-Dienstleister verfolgt. In diesem Ansatz liegt die Zuständigkeit für die Steuerung und Kontrolle der CM-Dienstleister sowie die interne Koordination bei Organisationseinheiten, welche auf ihrer Hierarchiestufe voneinander unabhängig agieren. Beide Unternehmen unterscheiden sich zudem in der Ausgestaltung der dezentralen Zuständigkeit. In Fallstudie 1 wird die Zuständigkeit für spezifische Management-Praktiken intern dezentral auf die beteiligten Fachabteilungen verteilt. In Fallstudie 4 werden innerhalb eines Organisationsbereiches länderspezifisch und produktgruppenspezifisch dezentrale Account Manager eingesetzt. Diese koordinieren die internen Stakeholder und wenden mit Unterstützung der Fachabteilungen Praktiken zur Steuerung und Kontrolle der CM-Dienstleister an. In Fallstudie 1 ist die Zuständigkeit für das Management der CM-Dienstleister aufgabenbezogen geregelt: Jeder Fachbereich ist für spezifische Praktiken wie Qualitäts-Audits oder Überprüfung der Termintreue zuständig. In Fallstudie 4 handelt es sich um eine Dienstleister-bezogene Zuständigkeit für alle anzuwendenden Management-Praktiken. In den Fallstudien mit dezentraler Zuständigkeit stellt die interne Transparenz über die eingesetzten Praktiken und aufgewendeten Ressourcen eine große Herausforderung dar.

In den Fallstudien 3 und 5 wird das Management von CM-Dienstleistern jeweils in strategische und operative Aufgabenbereiche unterteilt. Die strategischen Aufgabenbereiche werden in einer dedizierten Organisationseinheit zentral verantwortet. Diese setzt als Plattform unternehmensweite Standards für das Management der CM-Dienstleister und ist in strategische Projekte wie z. B. die Auswahl neuer CM-Dienstleister eingebunden. Für die operative Betreuung der CM-Dienstleister sind Account Manager zuständig, welche dezentrale Teams leiten und die Anwendung der Management-Praktiken für einen oder mehrere CM-Dienstleister verantworten. Die Teammitglieder sind formal den Fachabteilungen zugeordnet und übernehmen feste Rollen in den Teams.

\subsection{Implikationen für das Management von Contract- Manufacturing-Dienstleistern in der Supply Chain}

Basierend auf den Ergebnissen der Fallstudienanalysen lässt sich im Sinne des explorativen Forschungsdesigns ein Vorverständnis zur Beantwortung der Forschungsfragen formulieren. 
1. Welche Kontextelemente beeinflussen die situative Anwendung der Praktiken für das Management von CM-Dienstleistern?

Im Rahmen der Fallstudienanalyse konnten zehn relevante Kontextelemente identifiziert werden, welche die Anwendung spezifischer Management-Praktiken durch Unternehmen beeinflussen. Die Einbindung dieser Kontextelemente in die Steuerung und Kontrolle von CM-Dienstleistern erfolgt zweistufig: Basierend auf verschiedenen Kontextelementen werden in den betrachteten Unternehmen Management-Segmente definiert. Diese stellen die Grundausrichtung des Management-Ansatzes für die den Segmenten zugeordneten CM-Dienstleister dar. Bei Bedarf werden zusätzlich Management-Praktiken auf Basis spezifischer Kontextelemente eingesetzt.

Die erfassten Kontextelemente lassen sich, wie in Abb. 8.9 dargestellt, vier Kategorien zuordnen und verdeutlichen den interdisziplinären Charakter des Managements von CM-Dienstleistern. Die Relevanz produktbezogener Kontextelemente erfordert die Einbindung der Produktentwicklung während die logistischen Kontextelemente interne und extern operative Faktoren für die Anwendung von Management-Praktiken repräsentieren. Die geschäftsbezogenen Kontextelemente zeigen die Bedeutung auf strategischer Ebene getroffener Entscheidungen für die Steuerung und Kontrolle der Contract-Manufacturer auf. Aus der hohen Anzahl beziehungsbezogener Kontextelemente erwächst die Notwendigkeit der individuellen und engen Betreuung von CM-Dienstleistern.

Zur Beantwortung der ersten Forschungsfrage lässt sich ohne Anspruch auf Vollständigkeit der identifizierten Kontextelemente festhalten, dass sich der Anwendungskontext von Management-Praktiken zur Steuerung und Kontrolle von CM-Dienstleistern vertikal über alle Entscheidungsebenen des Supply Chain Managements erstreckt. Demnach sind diese Entscheidungsebenen im Management von Contract-Manufacturern in der Supply Chain entweder zu integrieren oder $\mathrm{zu}$ koordinieren. In diesem Zusammenhang wird auf Forschungsfrage 3 und die Zuständigkeit für das Management von CM-Dienstleistern verwiesen.

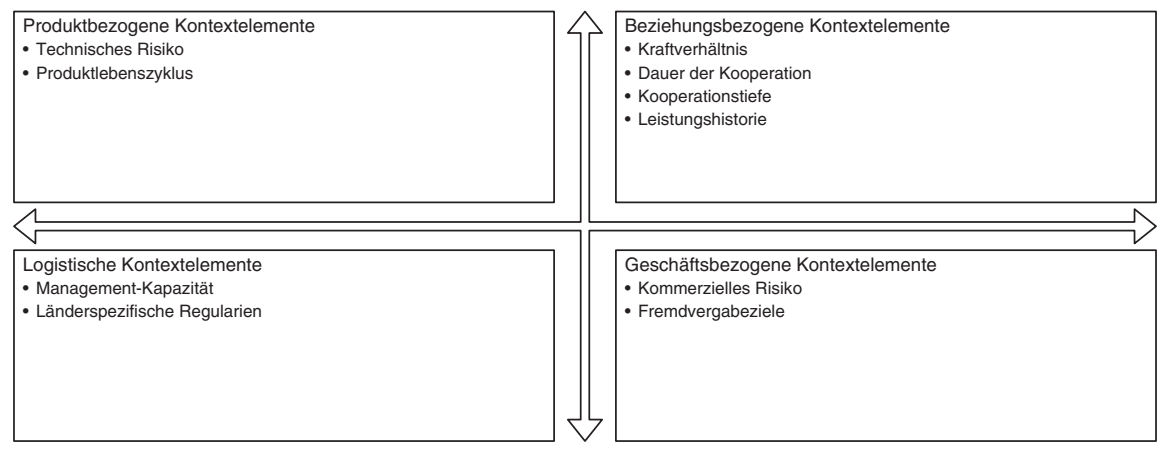

Abb. 8.9 Überblick relevanter Kontexteinflüsse 
2. Welche Management-Praktiken setzen Unternehmen situativ ein, um ihre CM-Dienstleister zu steuern und kontrollieren?

Aus den betrachteten Fallstudien lassen sich erste Erkenntnisse für die Beantwortung der zweiten Forschungsfrage ableiten. Es konnten übergreifende Management-Praktiken identifiziert werden, $\mathrm{zu}$ welchen in der operativen Anwendung vielfältige Gestaltungsvarianten existieren. Lediglich die kennzahlenbasierte Leistungskontrolle, Qualitäts-Audits und Incentivierungsmechanismen bilden eine fallstudienübergreifende, kontextunabhängige Basis von ManagementPraktiken. Zur zielgerichteten Allokation verfügbarer Management-Ressourcen wird die Mehrheit der identifizierten Management-Praktiken dienstleisterspezifisch genutzt. Darunter fallen auch fortgeschrittene Auditvarianten. Aufgrund des zunehmenden Digitalisierungs- und Automatisierungsgrades könnten sich digitale Kommunikationspraktiken in Zukunft als kontextunabhängiger Standard durchsetzen. Abb. 8.10 gibt einen Überblick über die identifizierten ManagementPraktiken.

Zur Beantwortung von Forschungsfrage zwei ist anzumerken, dass die Ergebnissteuerung und -kontrolle im Contract Manufacturing als Standard angesehen werden können. Zudem setzen Unternehmen verschiedene Praktiken zur Prozesskontrolle bei CM-Dienstleistern ein. Die Prozessteuerung ist weniger verbreitet, gewinnt bei Kommunikationsprozessen jedoch zunehmend an Bedeutung. Die soziale Steuerung von CM-Dienstleistern erfährt trotz der hohen Relevanz der Risikosteuerung derzeit nur geringe Aufmerksamkeit.

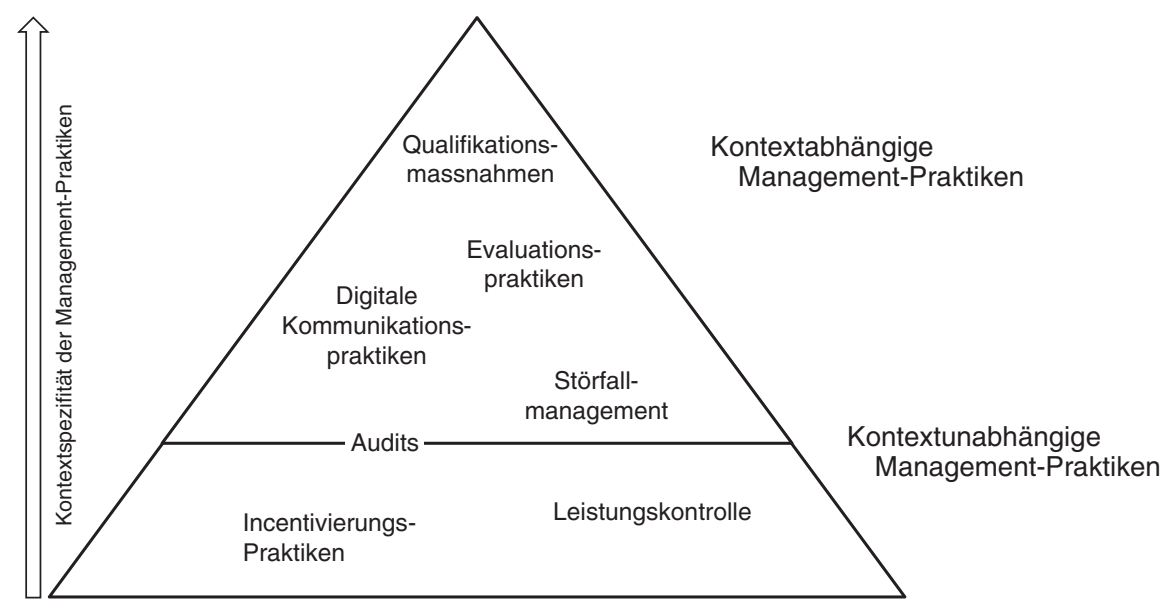

Abb. 8.10 Kontextspezifität der erfassten Management-Praktiken 
3. Wie koordinieren Unternehmen die Anwendung der Management-Praktiken zwischen den internen Stakeholdern?

Die Koordination der internen Stakeholder ist an die Gestaltung der Zuständigkeit für die Anwendung der Management-Praktiken im Unternehmen gebunden. Die unternehmensintern zuständige Instanz greift für die Durchführung der Management-Praktiken auf ExpertInnen aus relevanten Fachabteilungen zurück. Während der Durchführung werden sie durch die zuständige Instanz im Sinne einer temporären Projektorganisation angeleitet. Im hybriden Ansatz erfolgt eine aufgabengebundene Aufteilung der Zuständigkeiten: Um Zuständigkeitsüberschneidungen und -lücken zu vermeiden, sind die dezentralen Instanzen den zentralen Instanzen im hybriden Ansatz stets hierarchisch direkt untergeordnet. Die Verteilung der Zuständigkeit bringt in verschiedenen organisatorischen Ansätzen spezifische Vor- und Nachteile mit sich. So wirkt sich eine zentrale Instanz positiv auf die interne Transparenz und die Existenz von Standards hinsichtlich des Managements von CM-Dienstleistern aus. Dezentrale Instanzen ermöglichen hingegen eine enge fachliche Betreuung von CM-Dienstleistern. Unter Berücksichtigung der kontextrelevanten Entscheidungsebenen des Supply Chain Managements lässt sich hinsichtlich der organisatorischen Zuständigkeit folgender Zusammenhang identifizieren: Im dezentralen und hybriden Organisationsansatz werden die für den Anwendungskontext relevanten Entscheidungsebenen durch die zuständige Instanz koordiniert, während im zentralen Ansatz eine Integration der Entscheidungsebenen stattfindet. Für Unternehmen stellt sich daher die Frage nach den relevanten Typen von Kontextelementen ihrer Fremdvergabe, um den geeigneten organisatorischen Zuständigkeitsansatz zu identifizieren.

Die empirische Untersuchung des Managements von CM-Dienstleistern anhand der vorliegenden Fallstudien liefert erste Erkenntnisse darüber, wie Unternehmen der pharmazeutischen Industrie Praktiken zum Management von CMDienstleistern auswählen und durchführen. Es konnte aufgezeigt werden, welche Kontextelemente die Anwendung von Management-Praktiken beeinflussen und wie Unternehmen diese Kontextelemente in ihr Vorgehen integrieren (Forschungsfrage 1). Der situative Management-Ansatz von Unternehmen gegenüber ihren CM-Dienstleistern in der Praxis konnte anhand der identifizierten kontextunabhängigen und kontextabhängigen Management-Praktiken nachgewiesen werden. Als weiterer Beitrag zur Beantwortung von Forschungsfrage 2 konnte eine Übersicht über in der Praxis genutzten Management-Praktiken gegeben werden. Zudem wurde im Zuge der Beantwortung von Forschungsfrage 3 dargelegt, dass ausgewählte organisatorische Zuständigkeitsformen die Anwendung von Management-Praktiken in Unternehmen ermöglichen.

\subsection{Limitationen und weiterer Forschungsbedarf}

Der Beitrag liefert Erkenntnisse dazu, wie Unternehmen die Steuerung und Kontrolle von CM-Dienstleistern organisieren und welche konkreten Praktiken sie anwenden. Darüber hinaus konnte ein Beitrag zum Verständnis des situativen Managements von CM-Dienstleistern geliefert werden. 
Die Limitationen knüpfen am empirischen Design an, welches einen explorativen Ansatz mit sechs Fallstudien aus der pharmazeutischen Industrie enthält. Aufgrund des gewählten Fallstudienansatzes können kausale Beziehungen zwischen den Management-Praktiken und ihrem Anwendungskontext nicht identifiziert werden. Dem stehen die mit dem Fallstudienansatz induktiv gewonnenen Erkenntnisse gegenüber, welche für die Spezifikation tiefergehender Untersuchungen zu den Praktiken des Managements von CM-Dienstleistern dienen können. Die gewonnenen Erkenntnisse sind zudem branchenspezifisch zu bewerten und lassen sich nicht unreflektiert auf das Management von Contract Manufacturern in anderen Branchen übertragen.

Die Untersuchung zeichnet ein erstes Bild vom methodischen Vorgehen beim Management von CM-Dienstleistern und der operativen Organisation der Steuerungs- und Kontrollpraktiken. Um die Gründe für das Scheitern von Fertigungsfremdvergaben zu identifizieren, sollten Management-Ansätze von Fremdvergaben im Bereich der Fertigung untersucht und verglichen werden. $\mathrm{Zu}$ diesem Zweck ist es notwendig, den Anwendungskontext zu beleuchten. Ein validiertes, auf Kontextelementen basierendes Modell des Anwendungskontextes kann die vergleichende Analyse des Managements von Contract-Manufacturern ermöglichen und als Grundlage zur Beantwortung der Frage dienen, warum Fremdvergaben scheitern. Um Handlungsempfehlungen für das operative Management von CM-Dienstleistern abzuleiten, ist die kontextspezifische Eignung verschiedener Zuständigkeitsformen in Verbindung mit den eingesetzten Praktiken zu untersuchen. Dies führt zu Rückschlüssen, wo die erfolgskritischen Faktoren von externen Fertigungsvergaben in der operativen Steuerung zu verorten sind.

\section{Literatur}

Azadian, F., Murat, A., \& Chinnam, R. B. (2015). Integrated production and logistics planning: Contract manufacturing and choice of air/surface transportation. European Journal of Operational Research, 247(1), 113-123.

Boulaksil, Y., \& Fransoo, J. C. (2010). Implications of outsourcing on operations planning: Findings from the pharmaceutical industry. International Journal of Operations and Production Management, 30(10), 1059-1079.

Campagnolo, D., \& Camuffo, A. (2017). Ownership and location in the small domestic appliances industry: The De'Longhi case. In T. Pedersen, T. Devinney, L. Tihanyi, \& A. Camuffo (Hrsg.), Breaking up the global value chain: Opportunities and consequences (S. 3-27). Bingley: Emerald Publishing.

Cavinato, J. L. (1989). The logistics of contract manufacturing. International Journal of Physical Distribution \& Materials Management, 19(1), 13-20.

Denicolai, S., Strange, R., \& Zucchella, A. (2015). The dynamics of the outsourcing relationship. In R. Van Tulder, A. Verbeke, \& R. Drogendijk (Hrsg.), Progress in international business research (Bd. 10, S. 341-364). Bingley: Emerald Group Publishing Limited.

Eisenhardt, K. (1989). Building theories from case study research. The Academy of Management Review, 14(4), 532-550.

Foerstl, K., Kirchoff, J. F., \& Bals, L. (2016). Reshoring and insourcing: Drivers and future research directions. International Journal of Physical Distribution \& Logistics Management, 46(5), 492-515. 
Goh, S. H., \& Eldridge, S. (2015). New product introduction and supplier integration in sales and operations planning: Evidence from the Asia Pacific region. International Journal of Physical Distribution \& Logistics Management, 45(9-10), 861-886.

Grand View Research. (2017). Pharmaceutical Contract Manufacturing/Contract Research Market Report, 2025. https://www.grandviewresearch.com/industry-analysis/pharmaceuticalcontract-manufacturing-market. Zugegriffen: 13. Okt. 2020.

Gray, J. V., Tomlin, B., \& Roth, A. V. (2009). Outsourcing to a powerful contract manufacturer: The effect of learning-by-doing. Production and Operations Management, 18(5), 487-505.

Guzman, G. A. C. (2003). Inside modular production networks: 'soft operational issues' in building factory competitiveness. Integrated Manufacturing Systems, 14(1), 6-15.

Handley, S., \& Gray, J. (2015). Managing quality in a heterogeneous contract manufacturing environment. Decision Sciences, 46(6), 1011-1048.

Ibrahim, S. E., \& Altahawi, K. F. M. (2018). Opportunism and the dynamics of strategic outsourcing relationships. Journal of Global Operations and Strategic Sourcing, 11(2), 224-249.

Ivert, L. K., \& Jonsson, P. (2010). The potential benefits of advanced planning and scheduling systems in sales and operations planning. Industrial Management \& Data Systems, 110(5), 659-681.

Ivert, L. K., \& Jonsson, P. (2014). When should advance planning and scheduling systems be used in sales and operations planning? International Journal of Operations and Production Management, 34(10), 1338-1362.

Jakovcic, K. (2008). Contract manufacturing strategies: Market developments, technology transfer and key success factors. London: Business Insights.

Kaipia, R., \& Holmström, J. (2007). Selecting the right planning approach for a product. Supply Chain Management, 12(1), 2-13.

Kang, M., Wu, X., Hong, P., \& Park, Y. (2012). Aligning organizational control practices with competitive outsourcing performance. Journal of Business Research, 65(8), 1195-1201.

Kang, M., Wu, X., Hong, P., Park, K., \& Park, Y. (2014). The role of organizational control in outsourcing practices: An empirical study. Journal of Purchasing \& Supply Management, 20(3), 77-185.

Kim, B., Leung, J. M. Y. M., Park, K., Zhang, G., \& Lee, S. (2002). Configuring a manufacturing firm's supply network with multiple suppliers. IIE Transactions, 34(8), 663-677.

Kim, B. (2003). Dynamic outsourcing to contract manufacturers with different capabilities of reducing the supply cost. International Journal of Production Economics, 86(1), 63-80.

Kinkel, S. (2014). Future and impact of backshoring - Some conclusions from 15 years of research on German practices. Journal of Purchasing and Supply Management, 20(1), 63-65.

Mason, S. J., Cole, M. H., Ulrey, B. T., \& Yan, L. (2002). Improving electronics manufacturing supply chain agility through outsourcing. International Journal of Physical Distribution \& Logistics Management, 32(7), 610-620.

Mohammed, I. R., Shankar, R., \& Banwet, D. K. (2008). Creating flex-lean-agile value chain by outsourcing: An ISM-based interventional roadmap. Business Process Management Journal, 14(3), 338-389.

Oduro, S. (2019). Examining open innovation practices in low-tech SMEs: Insights from an emerging market. Journal of Science and Technology Policy Management, 10(3), 509-532.

Oke, A. (2005). A framework for analysing manufacturing flexibility. International Journal of Operations and Production Management, 25(10), 973-996.

Oke, A., \& Onwuegbuzie, H. (2013). Outsourcing, subcontracting-in and radical innovativeness. The moderating effect of manufacturing strategy. Journal of Manufacturing Technology Management, 24(4), 511-535.

Serel, D. A., Dada, M., \& Moskowitz, H. (2001). Sourcing decisions with capacity reservation contracts. European Journal of Operational Research, 131(3), 635-648.

Sharma, S., \& Modgil, S. (2015). Supply chain and total quality management framework design for business performance-case study evidence. Journal of Enterprise Information Management, 28(6), 905-930. 
Singh, R., Kumar, R., \& Kumar, P. (2016). Strategic issues in pharmaceutical supply chains: A review. International Journal of Pharmaceutical and Healthcare Marketing, 10(3), 234-257.

Singh Srai, J., \& Gregory, M. (2008). A supply network configuration perspective on international supply chain development. International Journal of Operations and Production Management, 28(5), 386-411.

Soon, Q. H., \& Udin, Z. M. (2011). Supply chain management from the perspective of value chain flexibility: An exploratory study. Journal of Manufacturing Technology Management, 22(4), 506-526.

Tsay, A. A., Gray, J. V., Noh, I. J., \& Mahoney, J. T. (2018). A review of production and operations management research on outsourcing in supply chains: Implications for the theory of the firm. Production and Operations Management, 27(7), 1177-1220.

van Weele, A. J., \& Eßig, M. (2017). Strategische Beschaffung: Grundlagen, Planung und Umsetzung eines integrierten Supply Management. Dordrecht: Springer.

Webster, M., Alder, C., \& Muhlemann, A. P. (1997). Subcontracting within the supply chain for electronics assembly manufacture. International Journal of Operations \& Production Management, 17(9), 827-841.

Yam, A. Y. K., Chan, M. F. S., \& Chung, W. W. C. (2007). Networked enterprise: A case study of implementing an information network system for global product development. International Journal, 14(3), 369-386.

Zhang, M., Pawar, K. S., Shah, J., \& Mehta, P. (2013). Evaluating outsourcing partners' capability: A case study from the pharmaceutical supply chain. Journal of Manufacturing Technology Management, 24(8), 1080-1101.

Zion Market Research. (2019). Electronics Contract Manufacturing Services Market by Process: Global Industry Perspective, Comprehensive Analysis, and Forecast 2018-2025. https:// www.zionmarketresearch.com/report/electronics-contract-manufacturing-services-market. Zugegriffen: 13. Okt. 2020. 